\title{
Streaming flow by oscillating bubbles: quantitative diagnostics via particle tracking velocimetry
}

\author{
Rocío Bolaños-Jiménez ${ }^{1}$, Massimiliano Rossi ${ }^{2}$, David Fernandez Rivas ${ }^{3}$, \\ Christian J. Kähler ${ }^{2}$ and Alvaro Marin ${ }^{4} \dagger$ \\ ${ }^{1}$ Área de Mecánica de Fluidos, Departamento de Ingeniería Mecánica y Minera, Universidad de Jaén, \\ Jaén, Spain \\ ${ }^{2}$ Institute for Fluid Mechanics and Aerodynamics, Bundeswehr University Munich, Germany \\ ${ }^{3}$ Mesoscale Chemical Systems Group, Mesa +, University of Twente, The Netherlands \\ ${ }^{4}$ Physics of Fluids, University of Twente, The Netherlands
}

(Received 13 September 2016; revised 5 April 2017; accepted 6 April 2017;

first published online 10 May 2017)

Oscillating microbubbles can be used as microscopic agents. Using external acoustic fields they are able to set the surrounding fluid into motion, erode surfaces and even to carry particles attached to their interfaces. Although the acoustic streaming flow that the bubble generates in its vicinity has been often observed, it has never been measured and quantitatively compared with the available theoretical models. The scarcity of quantitative data is partially due to the strong three-dimensional character of bubble-induced streaming flows, which demands advanced velocimetry techniques. In this work, we present quantitative measurements of the flow generated by single and pairs of acoustically excited sessile microbubbles using a three-dimensional particle tracking technique. Using this novel experimental approach we are able to obtain the bubble's resonant oscillating frequency, study the boundaries of the linear oscillation regime, give predictions on the flow strength and the shear in the surrounding surface and study the flow and the stability of a two-bubble system. Our results show that velocimetry techniques are a suitable tool to make diagnostics on the dynamics of acoustically excited microbubbles.

Key words: bubble dynamics, drops and bubbles, microfluidics

\section{Introduction}

Oscillating microbubbles can drive flow in microconfined systems (Marmottant \& Hilgenfeldt 2004), and manipulate particles (Wang, Jalikop \& Hilgenfeldt 2012), drugs or micro-organisms (Lajoinie et al. 2016), among many other applications. For this reason, they are excellent candidates to enhance control in miniaturized systems. 
When the system is vibrated at low or moderate energy, the bubble responds with linear oscillations. Such interfacial motion attenuates in the surrounding liquid and generates a secondary flow that has been often referred as 'steady streaming' (Riley 2001) or as simply acoustic streaming. Not only was the flow generated by oscillating microbubbles studied in depth by Marmottant \& Hilgenfeldt (2003, 2004), they also took advantage of the high stresses that can be generated in the vicinity of the bubble to induce the deformation and eventual lysis of vesicles for biological applications (Marmottant, Biben \& Hilgenfeldt 2008; Pommella et al. 2015).

At higher acoustic energies, the microbubble oscillations become nonlinear and smaller bubbles are emitted from the bubble's tip (Zijlstra et al. 2015). Such regimes greatly enhance sonochemical reactions when compared with conventional sonoreactors (Fernandez Rivas et al. 2010, 2012a, 2013b). The pressure oscillations produced are also able to generate microcavitation in the surroundings of the bubble, which can be used to remove undesirable elements (Fernandez Rivas et al. 2012b), but also cause erosion (Fernandez Rivas et al. 2013a; van Wijngaarden 2016).

Removing contamination from surfaces or cleaning inaccessible cavities can be achieved with ultrasound-driven microbubbles (Otto et al. 2011; Verhaagen \& Fernandez Rivas 2016; van Wijngaarden 2016). For example, the decontamination of medical instruments in hospitals and dental clinics is often preceded by a thorough ultrasonic cleaning. In endodontics, acoustically driven cavitation has been reported to improve root canal cleaning (Macedo et al. 2014). Metal workshops use benchtop ultrasonic baths to clean small mechanical components, whereas larger equipment such as turbines or industrial pipelines can be cleaned in dedicated larger baths, or with special transducers able to clean the hull of boats (Mazue et al. 2011). Also in the semiconductor industry, cleaning smaller features on microdevice surfaces in cleanroom environments or other high-tech settings (Kim et al. 2010; Hauptmann et al. 2013; Brems et al. 2014) has become a very relevant and economic challenge.

It is now accepted that standard ultrasonic cleaning is mainly driven by jets formed during bubble collapse close to a solid surface, but the role of shockwaves cannot be neglected (van Wijngaarden 2016). Dijkink \& Ohl (2008) obtained careful measurements of the pressure and shear stress exerted by a jet on a wall, yielding maximum values of $3.5 \mathrm{kPa}$. Such shear stress occurs only during a few microseconds while the bubble spreads over the surface. Despite the jet's short life, the value of the instantaneous stress is enough to cause damage even to metallic surfaces. For other applications, such as the removal of biofilms, with typical elastic modulus of $10^{2} \mathrm{~Pa}$ and shear strength of approximately 10 Pa (Flemming, Wingender \& Szewzyk 2011), it is desirable to achieve a more steady and controlled flow, even if this implies a substantially lower shear rate (Verhaagen 2012; Verhaagen \& Fernandez Rivas 2016). Such acoustic streaming flow induced by an oscillating bubble has been also used to propel microparticles (Bertin et al. 2015) or even milimetric objects (Dijkink et al. 2006).

Although there have been a number of theoretical studies on the fluid dynamics of the bubble streaming regime (Longuet-Higgins 1998; Marmottant \& Hilgenfeldt 2003; Marmottant et al. 2006, 2008), experimental data are scarce. One of the main difficulties in studying the flow field experimentally is its three-dimensional nature. This was eventually solved by using side view visualization, but in most systems this is not possible due to the limited visual access. Another difficulty is the tendency of microparticles used as flow tracers to become trapped within the hydrodynamic vortices, which ruins the flow traceability. Such an effect can be reduced by using smaller particles (typically in the micron or submicron range), which require stronger optical magnification and consequently stronger illumination. 
Despite the large number of studies and applications developed in recent years, there are several fundamental questions that still need to be answered. Even for the simplest case of moderate-amplitude oscillations, accurate models on the dynamic response of a gas pocket under an acoustic field are scarce. Such models are able to predict the frequency at which the bubble's momentum is best transferred into the fluid, in order to save energy and improve the efficiency of the system. Miller \& Nyborg (1983) obtained approximate analytical expressions for the lowest resonance frequency of a bubble trapped in a pore. More recently Gelderblom et al. (2012) developed a more general model to obtain the resonant frequency of a bubble performing small oscillations. Using level set methods to track the bubble's interface, Stricker (2013) analysed the bubble oscillations when subjected to large forced amplitudes, obtaining similar resonances and behaviour as found analytically by Gelderblom et al. (2012).

In this work, we quantitatively characterize the streaming flow generated by an oscillating microbubble attached to an artificial crevice. Such data can be used to obtain detailed information on the bubble dynamics by making use of theoretical models. Due to the complexity of the flow, only qualitative comparisons of particle trajectories with the predicted streamlines have been performed so far, only partially confirming the validity of the models. However, in our work, the quantitatively resolved flow field is employed to obtain the bubble's resonant frequency, study its response to the energy delivered to the system, find the limits of the linear oscillation regime and to observe its performance in a two-bubble system. To achieve these aims, we rely on well-known analytical models that reproduce the observed fluid flow in very good approximation. Our results show that flow measurements can be used as diagnostics for the bubble dynamics which could additionally lead to optimization of the energy delivered to the system in real applications (Fernandez Rivas et al. 2010; Verhaagen 2012; Lajoinie et al. 2016). The precise determination of the different bubble oscillation regimes (linear, nonlinear, bubble emission) could allow for considerable energy savings, and a significant enhancement in control and reliability of processes.

The paper is organized as follows. In $\S 2$, the experimental set-up and techniques are described. In $\S 3$ the results corresponding to a single bubble are presented, the case study in $\S 3.1$, the effect of the frequency in $\S 3.2$ and the effect of the voltage in $\S 3.3$. The shear rate in the vicinity of the bubble is reported in $\S 4$. The results corresponding to a two-bubble system are described in $\S 5$. Finally, $\S 6$ is devoted to conclusions.

\section{Experimental set-up and techniques}

\subsection{Experimental set-up}

Air bubbles are spontaneously trapped in artificial crevices micromachined in silicon substrates (Fernandez Rivas et al. 2010; Zijlstra et al. 2015). The artificial crevices (or pits) are generated by plasma dry etching on silicon substrates and have a cylindrical shape with $30 \mu \mathrm{m}$ diameter and $10 \mu \mathrm{m}$ in depth (see figure 1).

The silicon substrates (chips) containing the pits are square shaped, $10 \mathrm{~mm}$ side and $500 \mu \mathrm{m}$ thick. Different pit arrangements can be micromachined in the substrates. For the study of individual bubbles, we used a substrate with 161 pits arranged in a honeycomb pattern separated by a distance of $500 \mu \mathrm{m}$. This inter-pit distance was typically enough to avoid interactions among bubbles, as we will discuss below. In order to study the interaction between two bubbles $(\$ 5)$ we employed a different arrangement in which two individual pits were separated a distance of $100 \mu \mathrm{m}$. 


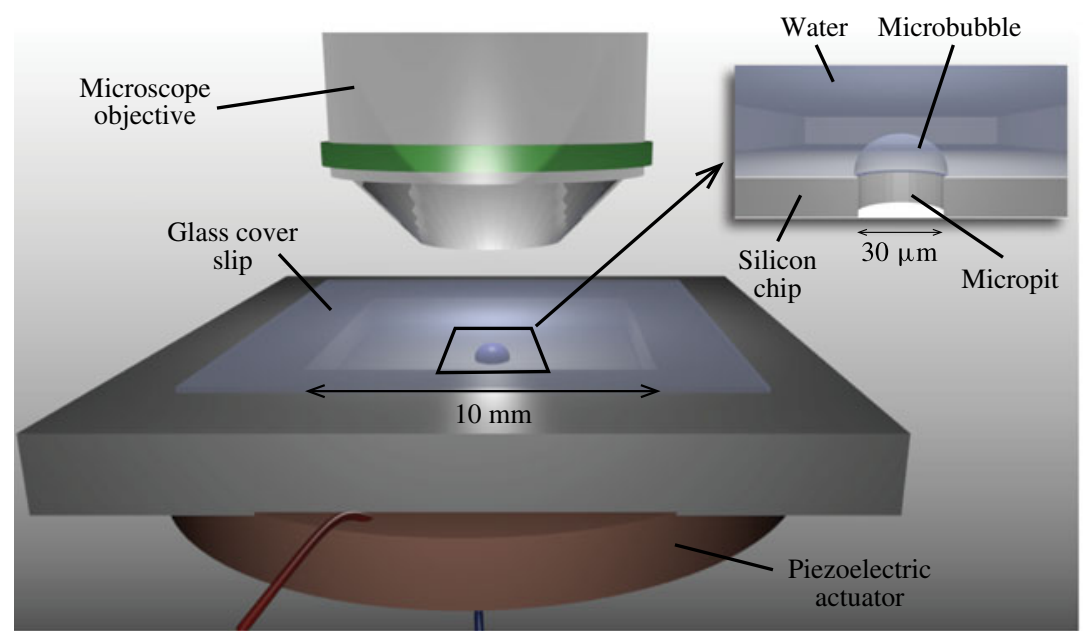

FIGURE 1. (Colour online) Experimental set-up employed to study microbubble streaming: microbubbles are trapped on artificially micromachined crevices (micropits) by plasma dry etching on a silicon substrate. The substrate is contained on an aluminium holder, covered with water and closed with a thin glass slide. Aluminium has been chosen for its high thermal conductivity and its efficiency dissipating heat. The glass slide permits visual access into the system using an upright microscope. A piezoelectric actuator attached to the bottom of the holder introduces acoustic vibrations into the system.

The chip was placed in an aluminium holder (see figure 1) which contained a rectangular chamber of $11 \times 11 \times 1 \mathrm{~mm}^{3}$ that permitted us to accommodate the chip and fill the chamber completely with ultra-purified water (Milli-Q). Once the chamber was filled with liquid, it was gently closed with a thin cover glass (Marienfeld No. $1.5,180 \mu \mathrm{m}$ thick), which allowed for optical access to the system.

In order to acoustically actuate the system, a cylindrical piezoelectric transducer (piezo) Ferroperm PZ27 (5 $\mathrm{mm}$ thick and $30 \mathrm{~mm}$ in diameter) was attached to the bottom of the holder (see figure 1). The attachment was done such that the distance from the piezo to the chip was only a few millimetres. To improve the coupling of the piezo with the aluminium holder, a layer of glycerine was applied in between. Piezo and holder were clamped together always in the same position in order to ensure reproducibility of the results.

Sinusoidal signals were applied to drive the piezo using a function generator (GW Instek AFG-2225) together with an amplifier (Krohn-Hite Model 7500). Both the frequency $f$, and the peak-to-peak voltage $V_{p p}$ of the signal actuating the piezo were measured using an oscilloscope (Tektronix TDS 2001c). In this work we study the frequency range $100 \mathrm{kHz} \leqslant f \leqslant 250 \mathrm{kHz}$ (close to the bubble's resonant frequency, see below) and amplitude range $30 \mathrm{~V} \leqslant V_{p p} \leqslant 300 \mathrm{~V}$ in order to analyse their effects on the streaming flow.

The acoustic energy input has been typically given as the power consumed by the piezo, calculated by calorimetric methods (Zeqiri 2007) or obtained experimentally by using hydrophones. Unfortunately, none of these options were possible in our case. On the one hand, the small size of the experimental system does not allow for hydrophones, on the other hand, our amplifier does not have control of the yielded power. Therefore, control on the input acoustic energy is made through the voltage 
delivered to the piezo, expressed in volts. However, a clear correlation of the input voltage with the acoustic energy will be discussed along the paper, in $\S 3.3$.

Visualization of the streaming flow is performed by a three-dimensional particle tracking velocimetry technique, which requires the use of fluorescent spherical microparticles (diameter $0.98 \mu \mathrm{m}$, from Microparticles $\mathrm{GmbH}$ ). A highly diluted solution of such particles was prepared using air-saturated ultrapure Milli-Q water and introduced in the chamber.

\subsection{Experimental techniques}

The particle trajectories and velocities are measured using astigmatism particle tracking velocimetry (APTV) (Cierpka et al. 2010; Rossi \& Kähler 2014). APTV is a single-camera particle tracking method in which an astigmatic aberration is introduced in the optical system by means of a cylindrical lens placed in front of the camera sensor. Consequently, an image of a spherical particle obtained in such a system shows a characteristic elliptical shape unequivocally related to its depth position $z$. The images of the particles in the microfluidic chip are taken using an upright microscope Zeiss Axio Imager in combination with a high-speed camera, the LaVision HS 4M pro imager, at recording speeds in the range from 1000 to $3000 \mathrm{fps}$. The optical arrangement consisted of a Zeiss LD Plan-Neofluar 40x/0.6 microscope objective lens and a cylindrical lens with focal length $f_{c y l}=300 \mathrm{~mm}$ placed in front of the camera sensor. Alternatively, a Zeiss EC Plan-Neofluar x40/0.75 objective with a larger aperture and shorter working distance was employed for those experiments in which the liquid volume in the chamber was intentionally diminished. Monodisperse spherical polystyrene particles with nominal diameters of $1 \mu \mathrm{m}$ were used for the experiments $\left(\rho_{p s}=1050 \mathrm{~kg} \mathrm{~m}^{-3}\right)$. The particles are fabricated and labelled with a proprietary fluorescent dye by Microparticles $\mathrm{GmbH}$ to be visualized with an epifluorescent microscopy system. Illumination is provided by a continuous diode-pumped laser with $2 \mathrm{~W}$ at $532 \mathrm{~nm}$ wavelength. This configuration provided a measurement volume of $600 \times 600 \times 120 \mu^{3}$ with an estimated uncertainty in the particle position determination of $\pm 1 \mu \mathrm{m}$ in the $z$-direction and less than $\pm 0.1 \mu \mathrm{m}$ in the $x$ - and $y$ directions. More details about the experimental configuration and uncertainty estimation of the APTV system can be found elsewhere (Rossi \& Kähler 2014).

\section{Characterization of the streaming flow for a single bubble}

In this section we aim at finding a method to evaluate the strength of the flow and connect it with the bubble's oscillations using some of the most accepted models in the literature. With this information, we can obtain the resonant frequency of oscillation by locating the frequency at which the flow strength is the highest. The characterization of the streaming flow around an oscillating microbubble of $30 \mu \mathrm{m}$ in diameter is done via particle tracking velocimetry for frequencies spanning from 100 to $250 \mathrm{kHz}$ (Fernandez Rivas et al. 2010, 2013b), first at a fixed peak-to-peak driving voltage of $30 \mathrm{~V}$, and then at a varying voltage in order to study its effect on the streaming flow.

As the system formed by the piezo, aluminium holder and chamber vibrates, it might reach resonances yielding higher input of acoustic energy into the system. This would be inconvenient since our aim is to quantify the streaming flow for different frequencies at fixed input energy. In order to confirm that the input energy is approximately constant, we measured the electrical current drawn by the piezo 
(a)
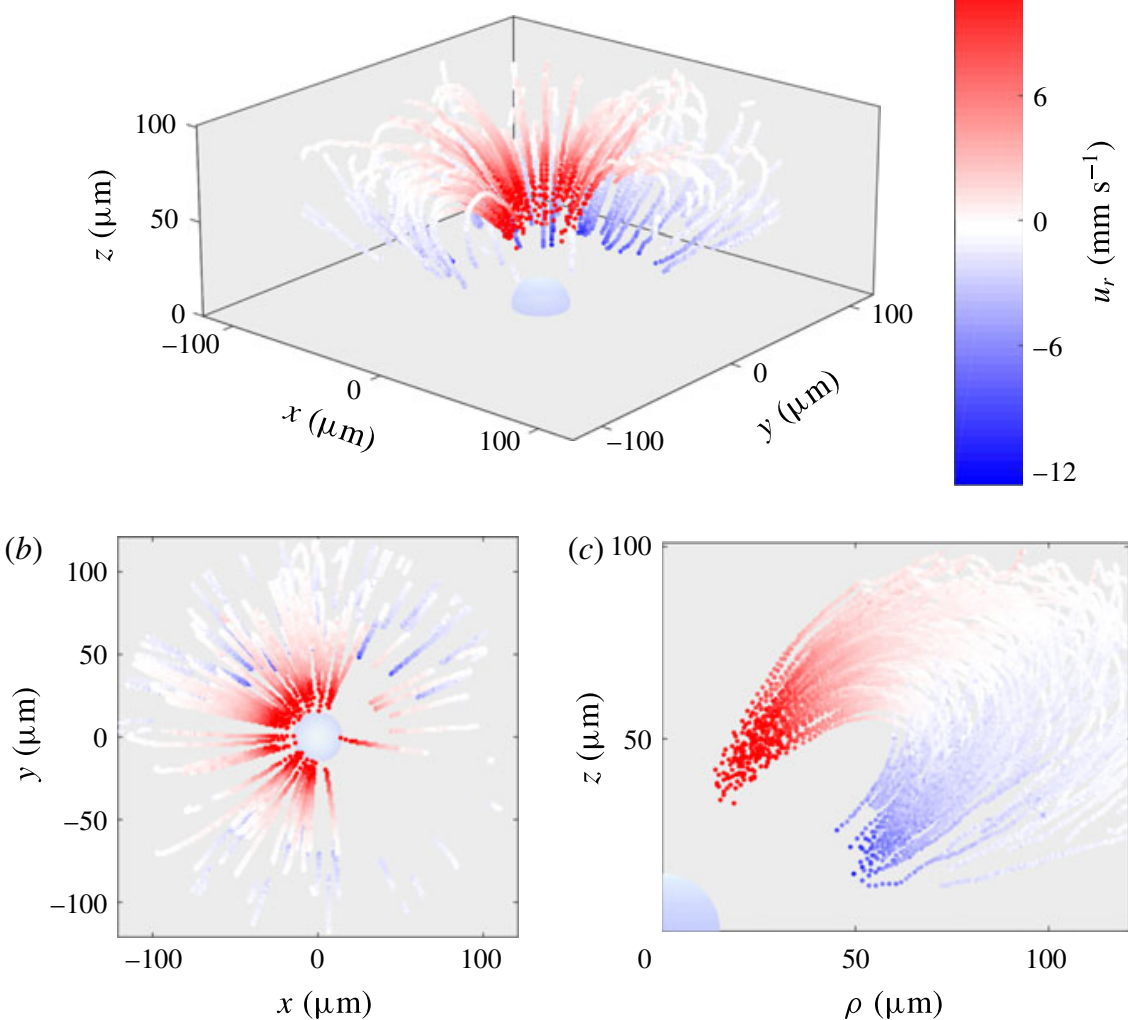

Figure 2. (Colour online) Particle trajectories for a bubble actuated at a frequency $f=150 \mathrm{kHz}$ with a driving voltage of $V_{p p}=30 \mathrm{~V}$ in different projections: $(a)(x, y, z)$ space, $(b)(x, y)$ plane and $(c)(\rho, z)$ plane, in cylindrical coordinates. Particles are coloured corresponding to their value of the (spherical) radial velocity component $u_{r}$. Positive values indicate particles moving away from the bubble and vice versa. A movie displaying the same data set is included in the supplementary materials.

in the frequency range of interest. The results of the frequency-voltage scan can be found in the supplementary material (available at https://doi.org/10.1017/jfm.2017.229) and confirm that the energy input does not change significantly for a fixed voltage input between 100 and $250 \mathrm{kHz}$ (below $10 \%$ of variation).

\subsection{Case study: $150 \mathrm{kHz}, 30 \mathrm{~V}$}

The case of $[f, V]=[150,30]$ on a chip with pits separated $500 \mu \mathrm{m}$ (corresponding to approximately 16 diameters) is chosen to illustrate the typical flow structure generated by an oscillating microbubble in the surrounding liquid. Since experiments are run with a low concentration of tracer particles, several repetitions are required to acquire significant statistics for each set of parameters. The particle trajectories shown in figure 2 correspond to several experiments and therefore different bubbles (approximately a dozen different ones in this particular case). The same data set is displayed in a movie available in the supplementary material.

Figure 2(a) corresponds to a three-dimensional view in the $(x, y, z)$ space of the bubble and particle trajectories, while figure $2(b)$ shows a projection in the 
$(x, y)$ space and 2(c) in the plane defined by the cylindrical coordinates $(\rho, z)$. Particles are alternatively attracted (blue) and repelled (red) from the bubble, following loop-like trajectories in planes that are perpendicular to the chip, and establishing a 'fountain-like' flow pattern, as has been classically described by Miller (1988) and Marmottant \& Hilgenfeldt (2003), among many others. The colour code in figure 2 corresponds to the radial component (in spherical coordinates) of the velocity field, $u_{r}$. Positive values $\left(u_{r}>0\right.$, red) indicate particles moving away from the bubble and negative values $\left(u_{r}<0\right.$, blue) indicate that they are approaching to it. Particles velocity increase steeply as they move closer to the bubble and slow down to almost negligible values far away from it. Such large dynamic range becomes an experimental difficulty for tracking particles: those in the vicinity to the bubble move too fast to be tracked under our choice of recording frame rate, while particles separated by three bubble radii hardly move. Such a large velocity gradient is common in microbubble streaming systems, even with cylindrical bubbles (Miller \& Nyborg 1983; Marmottant \& Hilgenfeldt 2003; Marin et al. 2015). Due to this particularity of the flow field, its quantification becomes a non-trivial task. In what follows, we propose a method to characterize the flow by obtaining a 'flow strength' magnitude using well-known models in the literature that will allow us to characterize the entire streaming flow for different driving parameters and make proper comparisons.

The clear symmetry around the $z$-axis shown by particle trajectories in figure $2(a, b)$ allows for a description of the flow in cylindrical coordinates $(\rho, z)$ (figure $2 c$ ).

The Reynolds number (based on the bubble radius) varies significantly from values $R e<10^{-2}$ at distances $\rho / a>2$, to $R e \sim 1$ in the close vicinity of the bubble interface. Our experimental method does not permit us to track the inertial behaviour of particles in the near field, nor is that the aim of this study. For more information on this last regime, we refer to Thameem, Rallabandi \& Hilgenfeldt (2016) who have recently studied the inertially driven migration of larger particles. In the following, we focus in the far-field region, where the flow field can be described as a Stokes flow using a streamfunction. Following the approach of Longuet-Higgins (1998), and using the method of images and singularity theory, the acoustic streaming flow can be obtained by assuming volumetric and translational oscillations of the bubble and applying the proper boundary conditions to obtain a streaming function. In this way, Marmottant \& Hilgenfeldt (2003) used the method of images to consider the presence of the wall and obtained the leading-order streamfunction in the far field:

$$
\psi=-\frac{A_{f}}{r} \cos ^{2}(\theta) \sin ^{2}(\theta),
$$

which accounts for the dipolar part (far field) of the full solution. $A_{f}$ is a constant, $r$ and $\theta$ are the distance from the bubble's centre and the elevation expressed in spherical coordinates. The streamlines given by (3.1) are plotted in figure 3, together with the particle trajectories obtained experimentally. Notice that the latter follows the streamlines obtained from (3.1) very closely, as can be observed in the figure. Deviations of the particle trajectories from the streamlines occur mainly in the regions where the streaming velocity is lower and Brownian motion dominates the particle dynamics. The components of the velocity field in spherical coordinates can be thus easily obtained:

$$
u_{r}(r, \theta)=-\frac{A_{f}}{r^{3}} \frac{\sin (2 \theta) \cos (2 \theta)}{\sin \theta}, \quad u_{\theta}(r, \theta)=-\frac{A_{f}}{r^{3}} \sin (\theta) \cos ^{2}(\theta) .
$$




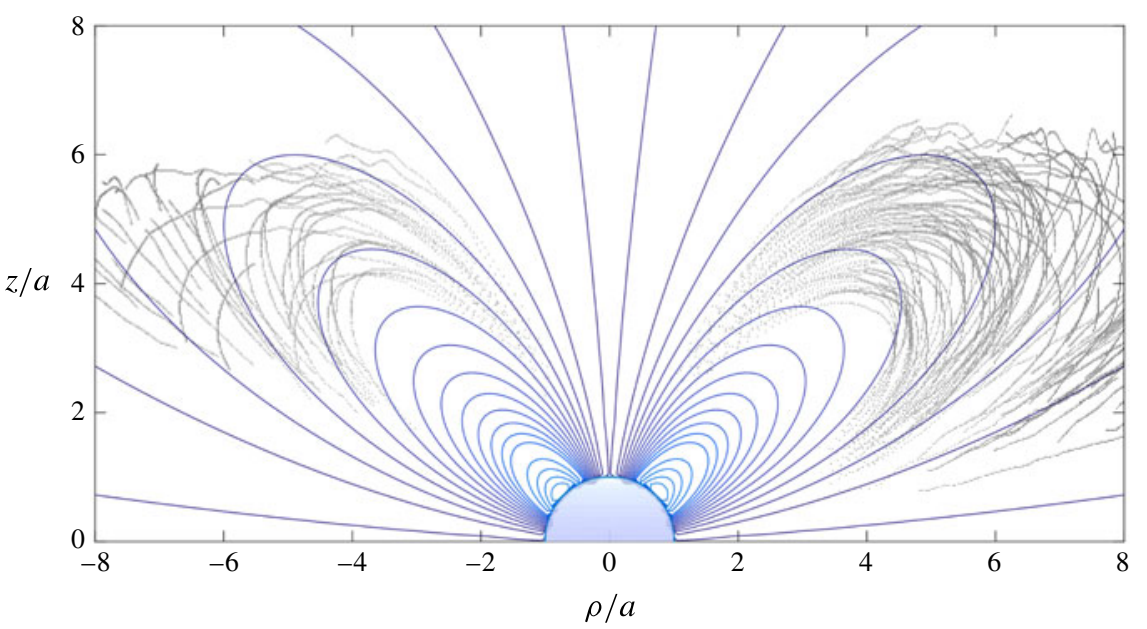

FIGURE 3. (Colour online) Qualitative comparison of model and experimental data. Blue lines represent streamlines from the dipolar term of the full streamfunction employed to experimentally characterize the flow strength (3.1). Black dotted lines represent experimental data from the case study $(150 \mathrm{kHz}, 30 \mathrm{~V})$. Deviations of the particle trajectories from the streamlines occur mainly in the regions where the streaming velocity is lower and Brownian motion dominates the particle dynamics. The data are projected in cylindrical coordinates, with $\rho$ radial distance and $z$ height, both normalized over the bubble radius $a$.

Our approach to assess the flow strength consists of using $A_{f}$ as a fitting parameter: for each experiment, we find the value of $A_{f}$ that better fits the measured velocity field using expressions (3.2). A functional form for $A_{f}$ was proposed by Marmottant \& Hilgenfeldt (2003) as

$$
A_{f}=B \epsilon^{2} a^{4} \omega
$$

where $\epsilon$ is the bubble's relative amplitude of oscillation, $a$ is the bubble's radius, $\omega$ the angular frequency $\omega=2 \pi f, f$ the driving frequency and $B$ is a constant that depends on the oscillation mode (Longuet-Higgins 1998; Marmottant et al. 2006). In a different configuration, Wang, Rallabandi \& Hilgenfeldt (2013) obtained the shape oscillations of a pinned cylindrical bubble at a wall and using an asymptotic model, they derived the frequency position and width of the bubble's resonance peaks, which compared qualitatively well with experimental data. In the present work however, it is assumed that the bubble is oscillating in a low resonance mode, as in Marmottant et al. (2006). Although our model is simpler than that used by Wang et al. (2013), the comparison shown here is strictly quantitative, as will be noticed later.

Thus, in our approach the flow strength is assessed by finding the value of $A_{f}$ (in a nonlinear least squares routine) that better fits the absolute velocity $|u|=\sqrt{u_{r}^{2}+u_{\theta}^{2}}$ with the functional form in (3.2). Note that the functional form employed for the velocity is only the dominant far-field term of the full streaming function, and therefore an approximation of the full one. This approximation is nonetheless justified since the majority of our measurements lay far from the bubble interface (a few bubble's diameters), as can be observed in figures $2-4$.

Figure 4 shows a comparison of the model with experimental data from the case under study $(150 \mathrm{kHz}, 30 \mathrm{~V})$. The data are plotted in cylindrical coordinates 
(a)

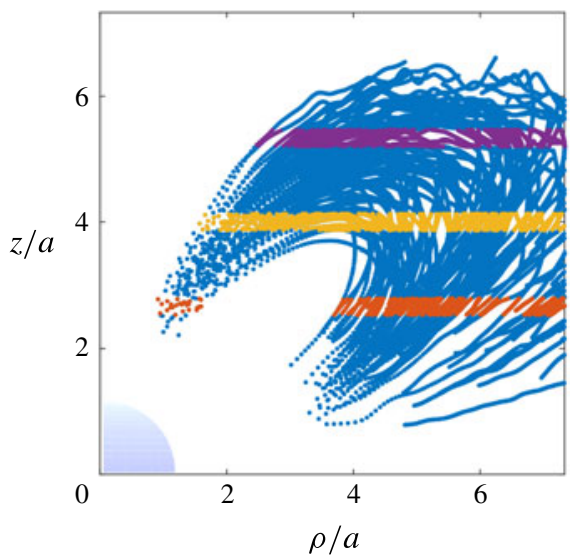

(b)

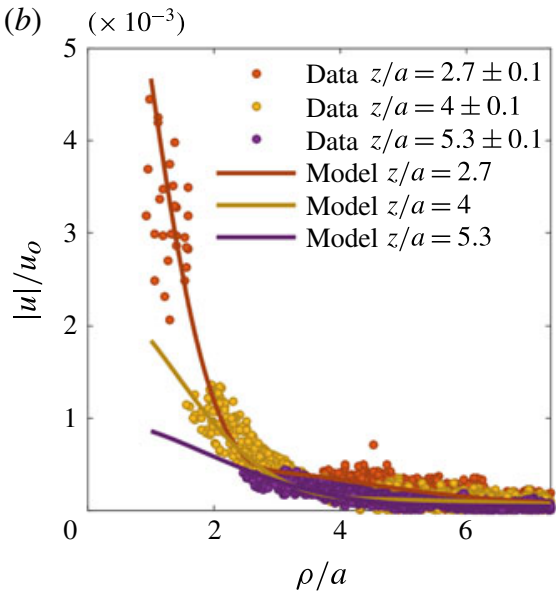

FIGURE 4. (Colour online) Quantitative comparison of the proposed fit from (3.2) with experimental data. (a) Particle trajectories used for the fit, plotted in cylindrical coordinates. (b) Comparison of the absolute velocity $|u|$ measured at different $z$ positions indicated in $(a)$ with the corresponding fit. Velocity values are normalized with $u_{o}=a \cdot f$. Although the model underestimates the velocities close to the bubble surface, it catches the overall trend of the velocity field. All data belong to the case of $f=150 \mathrm{kHz}$ and $V_{p p}=30 \mathrm{~V}$.

for convenience, where $\rho=r \sin (\theta)$ and $z=r \cos (\theta)$, and normalized with the bubble radius $a$. Figure $4(a)$ shows the positions where the particle velocity has been measured. Three $z$-positions have been selected and highlighted to make a clear quantitative comparison with the model. In figure $4(b)$ the predicted (lines) and measured (points) velocity values are plotted together for the three different $z$-positions that have been chosen as a function of the cylindrical radial position normalized with the bubble radius, $\rho / a$. The velocity is normalized using $u_{o}=a \cdot f$, which is proportional to the expected fluid velocity close to the bubble's surface $u_{s}=\epsilon^{2} a \omega$. Wang et al. (2013) as well as Marmottant et al. (2006) were able to achieve enough temporal and spatial resolution to measure the bubble's oscillations and give and independent and precise estimation of $\epsilon$. This is not the objective of the present manuscript and consequently we use $u_{o}$ instead of $u_{s}$ to normalize the velocities. In summary, the experimental data are well fitted with a single fitting parameter, the flow strength $A_{f}$, which in this particular case takes the value $A_{f}=5.7 \times 10^{8} \mu \mathrm{m}^{4} \mathrm{~s}^{-1}$.

As discussed above, most of the measured data lie in the range $\rho / a>2$, where the velocities are lower (typically $R e<10^{-2}$ ) and statistics are higher. Velocity increases rapidly as the particle approaches the bubble interface, reaching eventually $R e \sim 1$ if its streamline passes close enough to $\rho / a \sim 1$. Note that these regimes cannot be resolved using the current experimental settings and will not be discussed in this paper.

\subsection{Bubble's resonant frequency}

In this section we make use of the streaming flow velocity to find the bubble's resonant frequency in the range from 100 to $250 \mathrm{kHz}$, with a fixed driving voltage $\left(V_{p p}=30 \mathrm{~V}\right)$. The resonant frequency of the bubble oscillations is identified with the maximum in $A_{f}$, which we can find independently of the natural acoustic resonances of the experimental system. Since the flow structure observed within the frequency 


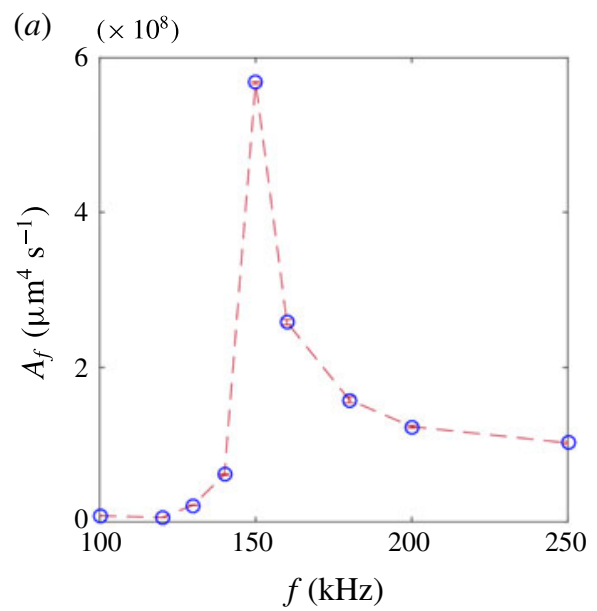

(b)

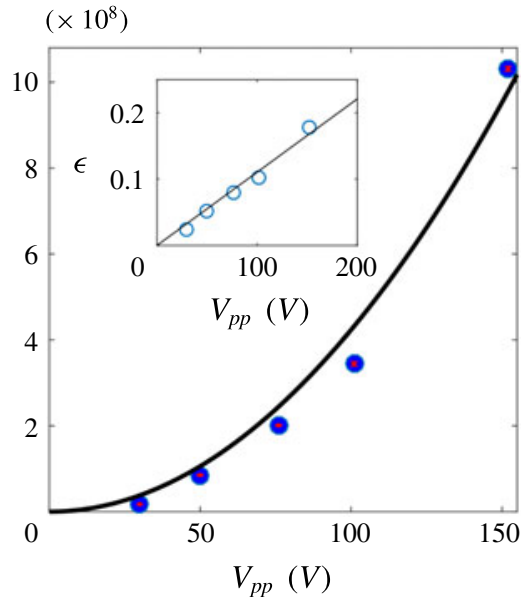

FIgURE 5. (Colour online) (a) Streaming flow strength $A_{f}$ found experimentally as a function of the driving frequency for a driving voltage $V_{p p}=30 \mathrm{~V}$. Dashed lines are a guide to the eye. $(b)$ Streaming flow strength $A_{f}$ as a function of the driving voltage for a driving frequency of $150 \mathrm{kHz}$. Circles represent experimental data and solid line is a fit of the form $A_{f} \propto V_{p p}^{2}$. The inset represents the relative oscillation amplitude $\epsilon$ (indirectly obtained via the experimental flow field, more details in the text) for different driving voltages. The solid line is a linear fit to the data.

range is similar to that shown in figure 2, the value of $A_{f}$ can be calculated as described in $\S 3.1$.

The experimental values obtained for $A_{f}$ are plotted in figure 5(a) for the range of frequencies explored. A clear resonant frequency can be observed at $f=150 \mathrm{kHz}$. Since no resonances have been found in the holder and chip system in this range of frequencies (see supplementary material), and $A_{f}$ depends quadratically on the bubble's amplitude of oscillation (see (3.3)), we can conclude that microbubbles in pits of $30 \mu \mathrm{m}$ diameter have a natural resonance at $f=150 \mathrm{kHz}$. Error bars based on the confidence bounds of the fitting are also plotted in figure 5, although difficult to see since the corresponding relative errors for $A_{f}$ are below $5 \%$. The coefficient of determination $R^{2}$ of the nonlinear fit gives valuable information on the fit, yielding values that go as high as 0.9 for $f=150 \mathrm{kHz}$ and as low as 0.3 for $f=100 \mathrm{kHz}$.

The resonant frequency value obtained experimentally can be compared with previous results in the literature. As a first approach, we can consider a free oscillating bubble immersed in an infinite volume of liquid in the case of small oscillations, which was discussed in the review by Plesset \& Prosperetti (1977). Following linearization of the pressure field, and using Rayleigh's bubble dynamic equation, an harmonic-oscillator type of equation with a natural resonance frequency takes the following general form:

$$
f_{o}=\frac{1}{2 \pi a} \sqrt{\frac{3 k P_{\infty} a-2 \gamma}{\rho a}},
$$

which has been written in terms of the bubble radius $a$, the liquid density $\rho$, its pressure at equilibrium $P_{\infty}$, the liquid's surface tension $\gamma$ and the process's polytropic exponent $k$. In the case of small bubbles excited in our frequency range, it can be 
assumed that the process is isothermal (see the argument of Plesset \& Prosperetti (1977)), and therefore $k \approx 1$. The first summand in expression (3.4) corresponds to the Minnaert frequency, while the second term introduces a correction due to surface tension effects. Under such assumptions, and using our experimental parameters, one can obtain a $f_{o}=182 \mathrm{kHz}$. That would be the expected result for a free bubble in a unbounded medium.

Miller \& Nyborg (1983) obtained an approximate analytical expressions for the lowest resonance frequency of a bubble trapped in a micropore (equation (25) in their paper), assuming a semi-spherical and clamped (or pinned) bubble oscillating isothermally:

$$
f_{m n}=\frac{1}{2 \pi a} \sqrt{\frac{15 k P_{\infty} a+120 \pi \gamma}{32 \rho a}},
$$

which in this case yields a value of $151.5 \mathrm{kHz}$, very close to our measured value.

Recent studies have attempted to refine the predictions with more sophisticated techniques. Gelderblom et al. (2012) used a more general approach than Miller \& Nyborg (1983) by assuming an arbitrary interface shape that was solved consistently in the limit of small amplitudes. Their results yield that the lowest resonant frequency for a pit in our same conditions should be close to $121 \mathrm{kHz}$. While Stricker (2013) found a value of $123.5 \mathrm{kHz}$ using level set simulations.

It is surprising that our results come closer to the somewhat simpler approach used by Miller \& Nyborg (1983). Nonetheless, no conclusions can be drawn on the suitability of the different models unless more data become available for different pit sizes under the same experimental conditions. Such a set of experiments goes beyond the scope of the present paper. A possible reason to explain this agreement/disagreement is the shape of the bubble interface at rest: although we cannot know its exact real shape in our current set-up, it was clear that it had a certain convex curvature due to the light intensity gradients along its interface. All models and simulations described above (Miller \& Nyborg 1983; Gelderblom et al. 2012; Stricker 2013) are however based on interfaces that are flat at rest. There is no literature to our knowledge dealing with the effect of the interface curvature on the bubble's oscillation resonances.

In order to validate our method, we can compare our result with that obtained by Marmottant et al. (2006) by direct imaging of the bubble's oscillations in a similar configuration. Using ultra-high-speed imaging to observe the oscillations of a bubble of $a=20 \mu \mathrm{m}$ at a driving frequency of $f=190 \mathrm{kHz}$, they obtained $\epsilon=0.077$ and $B=0.44$, which yields $A_{f} \approx 7.5 \times 10^{8} \mu \mathrm{m}^{4} \mathrm{~s}^{-1}$. The value lies very close to the $A_{f}$ obtained here using an experimental approach based on addressing the flow velocity, instead of the bubble's oscillations. Therefore, we conclude that our experimental method can be used to probe the bubbles dynamics in a quantitative manner with no need of direct observation of the bubble's oscillations, which typically requires both ultra-high speed and ultra-high magnification.

It is worth mentioning here that in the field of sonochemistry and ultrasonic cleaning (Fernandez Rivas et al. 2010, 2012b, 2013b) the working frequency is typically chosen in a different way, mostly by finding an acoustic resonance of the system in which the acoustic energy is more efficiently transferred. Such a strategy has been followed by Fernandez Rivas et al. (2010, 2013b) and Zijlstra et al. (2015) among others to induce nonlinear oscillations that will provoke the pinch-off of the main bubble into smaller ones. The sonochemical peak performance is typically achieved in such a regime, sometimes refereed as the high-power regime. Note that 
our experimental results have been obtained in the linear and harmonic-oscillations regime (low-power regime), and therefore they cannot be compared with those in sonochemistry. Nonetheless, using our experimental approach it is possible to discern easily and without high-speed imaging between linear and nonlinear oscillation regimes, as we will discuss in the next section.

\subsection{Streaming flow dependence on the driving voltage}

This section is dedicated to studying the response of the system to the driving voltage. For this set of experiments, we choose to maintain a constant driving frequency at its resonant value of $150 \mathrm{kHz}$ and vary the driving voltage in a certain range. The minimum value of the driving voltage is chosen according to the acquisition parameters, i.e. at that voltage at which particle velocities are measurable. For technical reasons, this series of experiments was performed with thicker chips, and therefore is expected to yield lower bubble excitation for the same driving voltage as was done in the previous subsection.

The maximum value of the driving voltage in the measurement range is connected with the transition into the nonlinear regime and thus it can provide interesting information about the bubble dynamics. The driving voltage range to study goes from $V_{p p}=30$ to approximately $300 \mathrm{~V}$. The particle trajectories within most of this regime are almost identical to those shown in figure 2 for $30 \mathrm{~V}$, and therefore the value of the flow strength $A_{f}$ can be calculated as described in $\S 3.1$.

The results are shown in figure $5(b)$, which shows a clear quadratic increase of the flow strength $A_{f}$ with the driving voltage. This is an expected result since the streaming flow, as a second-order effect, should scale with the oscillation amplitude as $u_{s} \propto \epsilon^{2}$, which at the same time scales linearly with the driving voltage, i.e. $\epsilon \propto V_{p p}$. With the obtained values of $A_{f}$, we can use (3.3) to give an indirect estimation of the relative bubble's amplitude of oscillation $\epsilon$ (taking $B=0.44$, as directly measured by Marmottant et al. (2006) for a similar configuration). The values obtained in this way for the relative bubble oscillations lay in the range that have been typically observed for bubbles in the linear oscillation regime, i.e. $\epsilon \lesssim 0.2$ (Marmottant et al. 2005; Garbin et al. 2007; Dollet et al. 2008). This is also consistent with our observations at higher voltages. For $V_{p p}>200 \mathrm{~V}$ the bubble becomes unstable, no steady flow pattern can be observed and therefore measurements were scarce. We interpret such a lack of steadiness in the flow as the transition into the nonlinear oscillation regime, in which the bubble stability is compromised and the generation of daughter bubbles is likely to occur (Stricker et al. 2013; Zijlstra et al. 2015). Such a dramatic change in the flow behaviour is a clear advantage of this experimental approach: the transition into the nonlinear regime can be easily found in this way since it is very reproducible for different experimental runs.

\section{Shear rate in the vicinity of the bubble}

In this section the shear or the strain rate of the streaming flow on the surface will be evaluated experimentally for voltages below the critical one to drive the bubble unstable $\left(V_{p p}<200 \mathrm{~V}\right)$. The aim is to study the cleaning capabilities of the streaming flow in the linear oscillations regime, where more control and reliability is achieved.

Since we have characterized the flow in the previous sections for different frequencies and voltages, we make use of the fittings found in $\S 3.3$ to obtain the flow strain on the chip's surface, defined as $\left|\partial u_{\rho} / \partial z\right|_{z=0}$, where $u_{\rho}$ is the cylindrical radial component of the velocity field. 


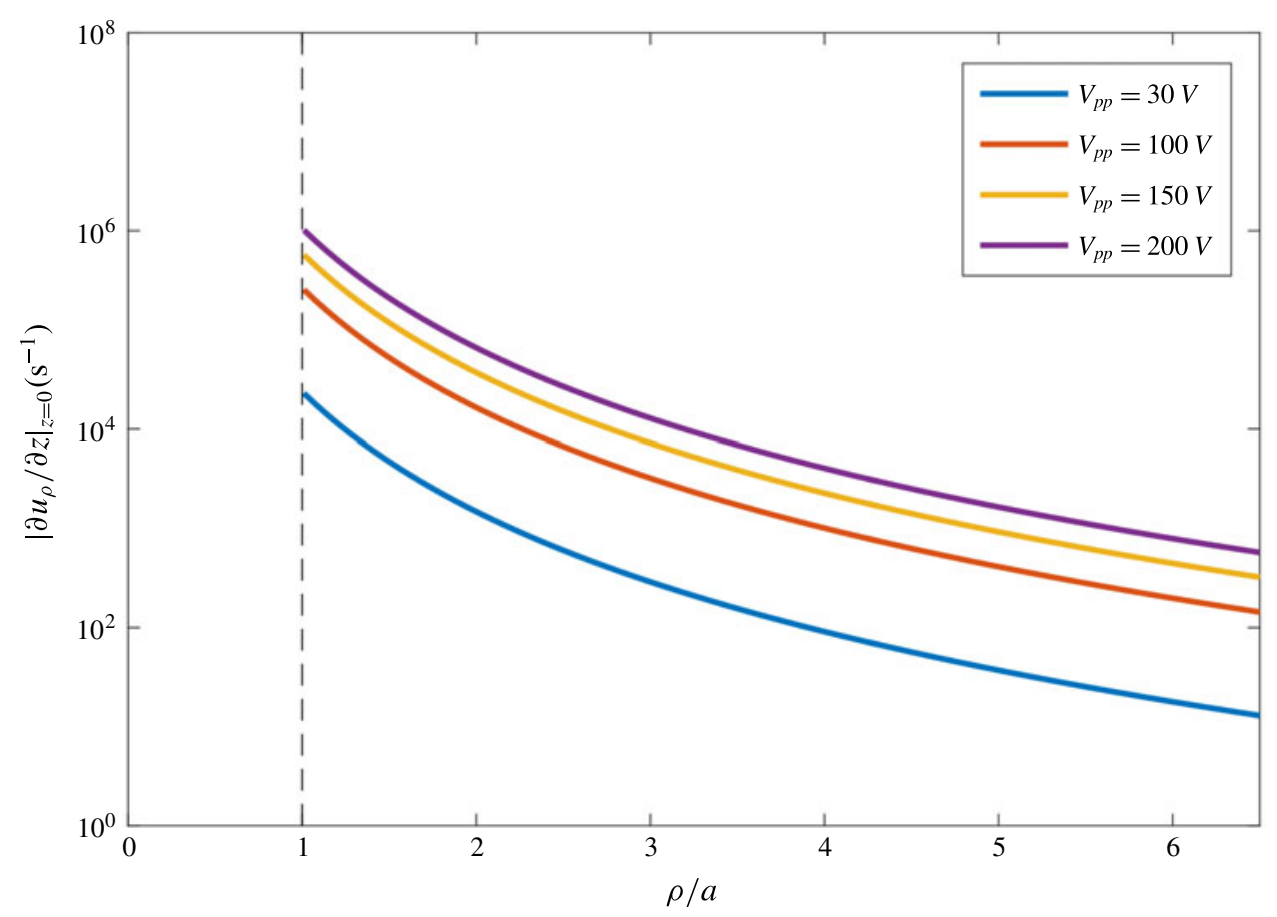

FIGURE 6. (Colour online) Strain rate over the chip's surface in the vicinity of the oscillating bubble, obtained from the experimental data fits in $\S 3$ for driving voltages from $30 \mathrm{~V}$ to $200 \mathrm{~V}$, and $f=150 \mathrm{kHz}$. The vertical striped line represents the bubble's interface. Note that the liquid employed is purified water $(\mu=1 \mathrm{mPa}$ s)

The result is shown in figure 6 for the different driving voltages employed in the experiments (from 30 to $200 \mathrm{~V}$ ). The data closest to the bubble's surface should be taken with care since we lack sufficient experimental data in that area, and the fitting is an approximation for the far field. According to (3.2), the shear rate increases rapidly as we approach the bubble's interface. Using the values of $A_{f}$ found experimentally, the obtained shear rate can be observed to increase with the driving voltage, as expected, and with the distance to the bubble surface. In particular, it can be noticed that it grows to relatively high values in an area of three bubble radii approximately, from $10^{3}$ up to $10^{6} \mathrm{~s}^{-1}$. Such values of shear rate are comparable to those reported for the hydrodynamic jets generated by collapsing bubbles close to surfaces (Dijkink \& Ohl 2008), which are thought to be responsible for cleaning in some applications. This indicates that acoustic cleaning applications could in principle be carried out under the linear oscillation regime, instead of the nonlinear one, reducing the energy consuming and possible surface damages, as well as enhancing the control of the cleaning process.

\section{Two-bubble system}

Practical applications typically rely on the flow generated by several bubbles instead of a single one. This can be achieved with artificially designed arrays of pits (Fernandez Rivas et al. 2010, 2013b; Verhaagen et al. 2016) or by spurious nucleation sites on a substrate or in the liquid bulk itself due to impurities or 
particles (Liger-Belair et al. 2002; Rodríguez-Rodríguez, Casado-Chacón \& Fuster 2014). However, bubbles in arrays or clouds interact in a non-trivial way. Particularly complex is the case of high-amplitude and nonlinear oscillations regime (high-power driving), where the bubble might eventually pinch off and emit smaller bubbles (Pelekasis et al. 1999; Fernandez Rivas et al. 2013b; Stricker et al. 2013). The case of two bubbles driven in the low-amplitude oscillations regime has been recently studied numerically by Doinikov \& Bouakaz (2016), revealing that larger velocities (and therefore larger wall shear stresses) should be expected as the bubbles are located closer to each other. The reason for the flow enhancement is due to a better coupling between the volume and translational oscillation modes, reflected through a change in the phase shift of each of the bubbles. However, note that the configuration studied by Doinikov \& Bouakaz (2016) is very different to ours: first, in their work, bubbles are freely suspended in the liquid (not attached to a wall). Secondly, bubbles respond to a plane travelling acoustic wave aligned with the bubbles' axis, so that the translational modes are also aligned with it. Instead, in our case the bubbles are attached to micropits, and the pressure field is mainly actuating perpendicularly to the substrate.

With the experimental data obtained in the previous sections for a single bubble, we are in an unique position to analyse the flow generated by two bubbles. For this purpose, we use a chip with two pits of the same diameter $(30 \mu \mathrm{m})$ and with their centres separated by a distance of $100 \mu \mathrm{m}$ (much smaller than in previous sections) with the aim of inducing the interaction. The driving frequency is kept at $150 \mathrm{kHz}$, the driving voltage is varied in the same range as in previous sections (from 30 to $300 \mathrm{~V}$ ), and the rest of experimental conditions are kept as in the previous sections.

Particle trajectories and velocities obtained in this system are shown for the particular case of $30 \mathrm{~V}$ in figure 7 . The particle trajectories are noticeably modified from the single-bubble case, especially in the area in between the bubbles. It can be observed that the range of the radial velocity increases compared with the corresponding single-bubble case (figure 2), which is a natural effect given that we have two sources of motion. Note that several particles trajectories actually cross the equidistant plane between the bubbles. The flow structure obtained for the rest of the driving voltages, although not shown here, is similar to that observed in figure 7 .

In order to make a fair comparison with the characterization obtained for a single bubble, and taking advantage of the Stokesian character of the flow at hand, we compute the flow field for the two-bubble system by simply adding up the solutions of two single bubbles separated a distance $d$ as:

$$
\boldsymbol{u}_{2 B}=\boldsymbol{u}(x, y, z)+\boldsymbol{u}(x, y+d, z),
$$

where $\boldsymbol{u}$ is derived from the streamfunction defined in (3.1) and $d=100 \mu \mathrm{m}$. Similarly to the procedure followed for a single bubble, by plotting the velocity magnitude as a function of the distance, the value of $A_{f}$ can be obtained as the fitting parameter. The results of the obtained flow strength $A_{f}$ are shown in figure 8 . The first thing to note is that the flow strength drops significantly for the two-bubble system at voltages $V_{p p}>100 \mathrm{~V}$. Experiments in that range of voltages were short since the bubbles became easily unstable, leaving their respective pits and merging together. Similar behaviour has been reported in previous studies related to cleaning purposes (Fernandez Rivas et al. 2013b; Zijlstra et al. 2015) for bubbles in the high-power 

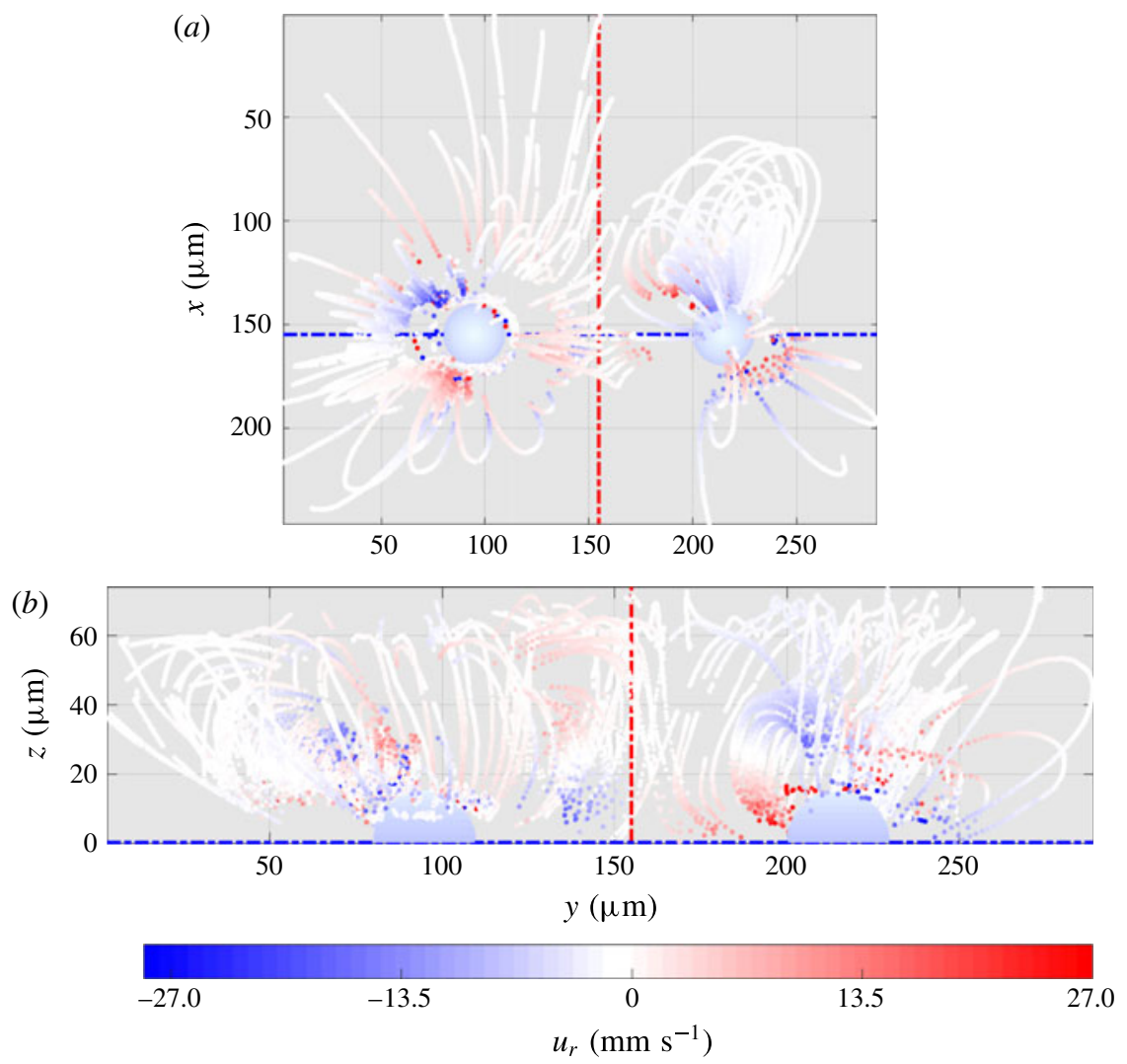

FIGURE 7. (Colour online) Double-bubble system driven at $30 \mathrm{~V}$, colour code corresponds to the value of the radial velocity value $u_{r}$. Panel $(a)$ shows the projection in the $(x, y)$ plane and $(b)$ the projection in the $(y, z)$ plane. The blue dashed line represents the axis joining the bubble centres, the red dashed line represents the equidistant plane between the bubbles. Note the larger velocity range when compared with that of a single bubble in figure 2.

regime. We believe that the reason for such instability are bubble-bubble attractive secondary Bjerknes forces, which may become significant at that acoustic energy and for that bubble-bubble separation. A similar merging effect has been observed for the same experimental system at larger voltages: when a certain threshold voltage is exceeded, clouds of smaller bubbles travel towards the midpoint of both, twoand three-pit bubble arrays (Fernandez Rivas et al. 2010, 2013b), which has been described analytically using Bjerkness forces (Stricker et al. 2013). Consequently, data points in that range of voltages are not taken into account for the calculation of the flow strength.

Secondly, it can be observed in figure 8 that the fitted function corresponding to the two-bubble system in the range of voltage with induces a clear steady flow is almost indistinguishable from that for the single-bubble system. This is an expected result since both single- and double-bubble systems are driven in the same way and under the same experimental parameters. Therefore, although higher velocities are obtained in the double-bubble system, the flow strength is not increased, contrary to the theoretical result obtained by Doinikov \& Bouakaz (2016). Consequently, no 


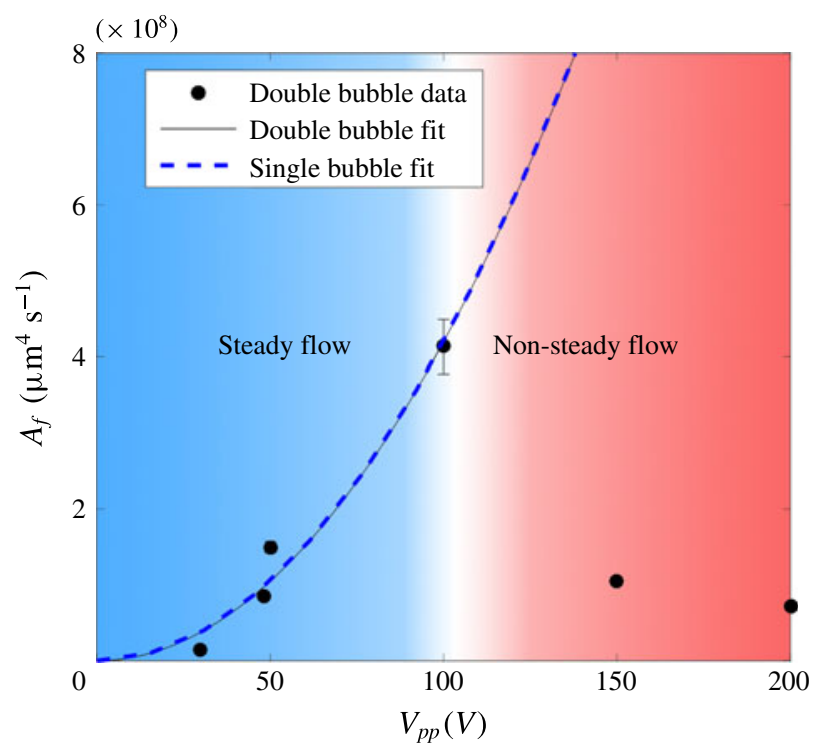

Figure 8. (Colour online) Comparison of the strength flow constant $A_{f}$ for the singlebubble system and the two-bubble system. The continuous and discontinuous lines are fits $A_{f} \propto V_{p p}^{2}$ for the two-bubble and the single-bubble system respectively.

significant flow enhancement has been observed in this particular configuration of two bubbles. Unfortunately, reproducing experimentally the configuration proposed by Doinikov \& Bouakaz (2016) is not straightforward and would require some type of optical bubble trap (Garbin et al. 2007). This is however far from the scope of the current paper.

\section{Conclusions}

In this work, we demonstrate a novel experimental approach to quantitatively characterize the streaming flow generated by sessile oscillating microbubbles and their intrinsic dynamics. In order to achieve this, we used a three-dimensional particle tracking velocimetry technique, namely astigmatic PTV (Rossi \& Kähler 2014), to measure the streaming flow generated by a sessile oscillating microbubble. Experimental data are then compared and fit using streaming function solutions. The fit is performed using a single parameter, $A_{f}$, which is directly proportional to the flow strength and is related to the bubble oscillations. This approach gives us a systematic, robust and unbiased quantification of the flow strength for a particular set of experimental parameters.

Following this approach, we have been able to:

(i) Find the bubble's lowest resonant frequency of oscillation by finding the largest flow strength parameter $A_{f}$. This is done in a fairly simple set-up, with no need for ultra-high-speed imaging or direct visualization of the bubble's interface. Such a resonant frequency is found to be different from the expected by recent theoretical and numerical predictions (Gelderblom et al. 2012; Stricker 2013). This disagreement could be due to the presence of certain curvature of the gas-liquid interface at rest. 
(ii) Find the maximum driving voltage in which the bubble is driven within the linear oscillation regime. We have found that the flow induced by bubbles oscillating at high amplitudes is characterized by an acute unsteadiness. Surprisingly, the transition from steady to unsteady flow is so sharp that it can be used to identify the transition from low to high oscillation amplitudes. This has been confirmed indirectly by comparing our measurements with ultra-high-speed observations from the literature.

(iii) Give educated guesses on the shear stress values in the vicinity of the bubble. We showed that the magnitude of shear stress values in proximity of the bubble are large enough to be used for cleaning purposes even in the linear regime. In comparison with the high-power, nonlinear regimes, the gentler linear regime provides higher reproducibility (as has been explicitly here shown), lower damage to the substrates, better temperature control and higher energy efficiency.

(iv) Analyse the flow structure in a two-bubble system and its threshold of stability. The transition to a non-steady flow in the two-bubble case occurs at a much lower voltage threshold, presumably due to bubble-bubble interactions, since the bubbles are often observed to jump from their crevices to merge once a certain critical driving voltage is reached.

Future work will aim to understand the apparent disagreement found in the predictions for the resonant frequency. As explained earlier, bubbles in artificial crevices typically show convex curved interfaces when observed from the outside and therefore the comparison with the models might be unfair. Up to now, the authors are not aware of any research dealing with the effect of curvature at rest of gas-liquid oscillating interfaces. The reasons for the observed bubble instability in the two-bubble case need to be further clarified in future studies. In practical applications it would be desirable to optimize the crevice density and therefore such bubble instability needs to be well understood. Finally, future studies could be done by using the same experimental approach shown here on bubble pits of different sizes, with enhanced control on the bubble interface (e.g. by pressurizing the liquid phase) and with more detailed observations of the bubble interface.

\section{Acknowledgements}

Technical and scientific support from R. Barnkob and A. Volk is specially acknowledged. D.F.R. and A.M. acknowledge previous unpublished work from X. M. Xu. R.B.J. acknowledges support from the Spanish mobility program for young doctors 'José Castillejo' 2014 (Spanish MECD). M.R., C.J.K. and A.M. acknowledge funding from German Research Council (Deutsche Forschungsgemeinschaft) via grant KA1808/17-1. Additionally, this paper is based upon work from COST Action MP1305, supported by COST (European Cooperation in Science and Technology).

\section{Supplementary material and movie}

Supplementary material and movie are available at https://doi.org/10.1017/jfm.2017.229.

\section{REFERENCES}

Bertin, N., Spelman, T. A., Stephan, O., Gredy, L., Bouriau, M., Lauga, E. \& Marmottant, P. 2015 Propulsion of bubble-based acoustic microswimmers. Phys. Rev. Appl. 4 (6), 064012. 
Brems, S., Hauptmann, M., Camerotto, E., Pacco, A., Kim, T. G., Xu, X., Wostyn, K., Mertens, P. \& De GendT, S. 2014 Nanoparticle removal with megasonics: a review. ECS J. Solid State Sci. Technol. 3 (1), N3010-N3015.

Cierpka, C., Rossi, M., Segura, R. \& Kähler, C. J. 2010 On the calibration of astigmatism particle tracking velocimetry for microflows. Meas. Sci. Technol. 22 (1), 015401.

DiJkink, R. \& OHL, C.-D. 2008 Measurement of cavitation induced wall shear stress. Appl. Phys. Lett. 93 (25), 254107.

Dijkink, R. J., VAn der Dennen, J. P., Ohl, C.-D. \& Prosperetti, A. 2006 The 'acoustic scallop': a bubble-powered actuator. J. Micromech. Microengng 16 (8), 1653-1659.

DoInikov, A. A. \& BouAKaZ, A. 2016 Microstreaming generated by two acoustically induced gas bubbles. J. Fluid Mech. 796, 318-339.

Dollet, B., Van Der Meer, S. M., Garbin, V., De Jong, N., Lohse, D. \& Versluis, M. 2008 Nonspherical oscillations of ultrasound contrast agent microbubbles. Ultrasound. Med. Biol. 34 (9), 1465-1473.

Fernandez Rivas, D., Ashokkumar, M., Leong, T., Yasui, K., Tuziuti, T., Kentish, S., Lohse, D. \& GARDEniers, H. J. G. E. $2012 a$ Sonoluminescence and sonochemiluminescence from a microreactor. Ultrason. Sonochem. 19 (6), 1252-1259.

Fernandez Rivas, D., Betjes, J., Verhaagen, B., Bouwhuis, W., Bor, T. C., Lohse, D. \& GARDENIERS, H. J. G. E. 2013a Erosion evolution in mono-crystalline silicon surfaces caused by acoustic cavitation bubbles. J. Appl. Phys. 113 (6), 064902.

Fernandez Rivas, D., Prosperetti, A., Zijlstra, A. G., Lohse, D. \& Gardeniers, H. J. G. E. 2010 Efficient sonochemistry through microbubbles generated with micromachined surfaces. Angew. Chem. Intl Ed. 49 (50), 9699-9701.

Fernandez Rivas, D., Stricker, L., Zijlstra, A. G., Gardeniers, H. J. G. E., Lohse, D. \& Prosperetti, A. $2013 b$ Ultrasound artificially nucleated bubbles and their sonochemical radical production. Ultrason. Sonochem. 20 (1), 510-524.

Fernandez Rivas, D., Verhahgen, B., Seddon, J. R. T., Zijlstra, A. G, Jiang, L.-M., van der Sluis, L. W. M., Versluis, M., Lohse, D. \& Gardeniers, H. J. G. E. $2012 b$ Localized removal of layers of metal, polymer, or biomaterial by ultrasound cavitation bubbles. Biomicrofluidics 6 (3), 034114.

Flemming, H.-C., Wingender, J. \& Szewzyk, U. 2011 Biofilm Highlights. vol. 5. Springer.

Garbin, V., Cojoc, D., Ferrari, E., Di Fabrizio, E., Overvelde, M. L. J., Van Der Meer, S. M., De Jong, N., Lohse, D. \& Versluis, M. 2007 Changes in microbubble dynamics near a boundary revealed by combined optical micromanipulation and high-speed imaging. Appl. Phys. Lett. 90 (11), 114103.

Gelderblom, H., Zijlstra, A. G., van Wijngaarden, L. \& Prosperetti, A. 2012 Oscillations of a gas pocket on a liquid-covered solid surface. Phys. Fluids 24 (12), 122101.

Hauptmann, M., Struyf, H., De Gendt, S., Glorieux, C. \& Brems, S. 2013 Importance of bubble size control in ultrasonic surface cleaning by pulsed high-frequency sound fields. ECS J. Solid State Sci. Technol. 3 (1), N3032-N3040.

KIM, W., PARK, K., OH, J., ChOI, J. \& Kim, H.-Y. 2010 Visualization and minimization of disruptive bubble behavior in ultrasonic field. Ultrasonics 50 (8), 798-802.

Lajoinie, G., De Cock, I., Coussios, C. C., Lentacker, I., Le Gac, S., Stride, E. \& VERSLUis, M. 2016 In vitro methods to study bubble-cell interactions: fundamentals and therapeutic applications. Biomicrofluidics 10 (1), 011501.

Liger-Belair, G., Vignes-Adler, M., Voisin, C., Robillard, B. \& Jeandet, P. 2002 Kinetics of gas discharging in a glass of champagne: the role of nucleation sites. Langmuir 18 (4), 1294-1301.

Longuet-Higgins, M. S. 1998 Viscous streaming from an oscillating spherical bubble. Proc. R. Soc. Lond. A 454 (1970), 725-742.

Macedo, R. G., Verhatgen, B., Fernandez Rivas, D., Gardeniers, J. G. E., van der Sluis, L. W. M., WesselinK, P. R. \& Versluis, M. 2014 Sonochemical and high-speed 
optical characterization of cavitation generated by an ultrasonically oscillating dental file in root canal models. Ultrason. Sonochem. 21 (1), 324-335.

Marin, A., Rossi, M., Rallabandi, B., Wang, C., Hilgenfeldt, S. \& Kähler, C. J. 2015 Three-dimensional phenomena in microbubble acoustic version. Phys. Rev. Appl. 3 (4), 041001.

Marmottant, P., Biben, T. \& Hilgenfeldt, S. 2008 Deformation and rupture of lipid vesicles in the strong shear flow generated by ultrasound-driven microbubbles. Proc. Natl Acad. Sci. USA 464 (2095), 1781-1800.

Marmottant, P. \& Hilgenfeldt, S. 2003 Controlled vesicle deformation and lysis by single oscillating bubbles. Nature 423 (6936), 153-156.

Marmottant, P. \& Hilgenfeldt, S. 2004 A bubble-driven microfluidic transport element for bioengineering. Proc. Natl Acad. Sci. USA 101 (26), 9523-9527.

Marmottant, P., van der Meer, S., Emmer, M., Versluis, M., De Jong, N., Hilgenfeldt, S. \& LohSE, D. 2005 A model for large amplitude oscillations of coated bubbles accounting for buckling and rupture. J. Acoust. Soc. Am. 118 (6), 3499-3505.

Marmottant, P., Versluis, M., De Jong, N., Hilgenfeldt, S. \& Lohse, D. 2006 High-speed imaging of an ultrasound-driven bubble in contact with a wall: 'Narcissus' effect and resolved acoustic streaming. Exp. Fluids 41 (2), 147-153.

Mazue, G., Viennet, R., Hihn, J.-Y., Carpentier, L., Devidal, P. \& Albaïna, I. 2011 Largescale ultrasonic cleaning system: design of a multi-transducer device for boat cleaning $(20 \mathrm{kHz})$. Ultrason. Sonochem. 18 (4), 895-900.

Miller, D. L. 1988 Particle gathering and microstreaming near ultrasonically activated gas-filled micropores. J. Acoust. Soc. Am. 84 (4), 1378-1387.

Miller, D. L. \& NyBORG, W. L. 1983 Theoretical investigation of the response of gas-filled micropores and cavitation nuclei to ultrasound. J. Acoust. Soc. Am. 73 (5), 1537-1544.

Otto, C., Zahn, S., Rost, F., Zahn, P., Jaros, D. \& Rohm, H. 2011 Physical methods for cleaning and disinfection of surfaces. Food Engng Rev. 3 (3-4), 171-188.

Pelekasis, N. A., Gaki, A., Doinikov, A. \& Tsamopoulos, J. A. 1999 Secondary Bjerknes forces between two bubbles and the phenomenon of acoustic streamers. J. Fluid Mech. 500, 313-347.

Plesset, M. S. \& Prosperetti, A. 1977 Bubble dynamics and cavitation. Annu. Rev. Fluid Mech. 9, $145-185$.

Pommella, A., Brooks, N. J., Seddon, J. M. \& Garbin, V. 2015 Selective flow-induced vesicle rupture to sort by membrane mechanical properties. Sci. Rep. 5, 13163.

Riley, N. 2001 Steady streaming. Annu. Rev. Fluid Mech. 33 (1), 43-65.

Rodríguez-Rodríguez, J., CASAdo-Chacón, A. \& Fuster, D. 2014 Physics of beer tapping. Phys. Rev. Lett. 113 (21), 214501.

Rossi, M. \& KäHLER, C. J. 2014 Optimization of astigmatic particle tracking velocimeters. Exp. Fluids 55 (9), 1-13.

STRICKER, L. 2013 Acoustic cavitation and sonochemistry. PhD thesis, University of Twente, Enschede.

Stricker, L., Dollet, B., Fernandez Rivas, D. \& Lohse, D. 2013 Interacting bubble clouds and their sonochemical production. J. Acoust. Soc. Am. 134 (3), 1854-1862.

Thameem, R., Rallabandi, B. \& Hilgenfeldt, S. 2016 Particle migration and sorting in microbubble streaming flows. Biomicrofluidics 10 (1), 014124.

VerhaAgen, B. 2012 Root canal cleaning through cavitation and microstreaming. PhD thesis, University of Twente, Enschede.

Verhatgen, B. \& Fernandez Rivas, D. 2016 Measuring cavitation and its cleaning effect. Ultrason. Sonochem. 29, 619-628.

Verhahgen, B., Liu, Y., Pérez, A. G., Castro-Hernández, E. \& Fernandez Rivas, D. 2016 Scaled-up sonochemical microreactor with increased efficiency and reproducibility. Chem. Select 1 (2), 136-139. 
Wang, C., Jalikop, S. V. \& Hilgenfeldt, S. 2012 Efficient manipulation of microparticles in bubble streaming flows. Biomicrofluidics 6 (1), 012801.

Wang, C., Rallabandi, B.\& Hilgenfeldt, S. 2013 Frequency dependence and frequency control of microbubble streaming flows. Phys. Fluids 25 (2), 022002.

VAN WiJngaARDEN, L. 2016 Mechanics of collapsing cavitation bubbles. Ultrason. Sonochem. 29 (C), 524-527.

ZeqIRI, B. 2007 Metrology for ultrasonic applications. Prog. Biophys. Mol. Biol. 93 (1), 138-152.

Zijlstra, A., Fernandez Rivas, D., Gardeniers, H. J. G. E., Versluis, M. \& Lohse, D. 2015 Enhancing acoustic cavitation using artificial crevice bubbles. Ultrasonics 56, 512-523. 